\title{
Monte Carlo Estimation of Absorbed Dose Distributions Obtained from Heterogeneous ${ }^{106}$ Ru Eye Plaques
}

\author{
Francisco J. Zaragoza ${ }^{a}$ Marion Eichmann ${ }^{b}$ Dirk Flühs ${ }^{a}$ Wolfgang Sauerwein ${ }^{a}$ \\ Lorenzo Brualla ${ }^{a}$ \\ ${ }^{a}$ NCTeam, Strahlenklinik, Universitätsklinikum Essen, Essen, and b Fakultät Physik, Technische Universität Dortmund, \\ Dortmund, Germany
}

\section{Keywords}

Brachytherapy · Beta emitter · Ruthenium - Eye plaques .

Simulation · Monte Carlo simulation · Dosimetry

\begin{abstract}
Background: The distribution of the emitter substance in ${ }^{106}$ Ru eye plaques is usually assumed to be homogeneous for treatment planning purposes. However, this distribution is never homogeneous, and it widely differs from plaque to plaque due to manufacturing factors. Methods: By Monte Carlo simulation of radiation transport, we study the absorbed dose distribution obtained from the specific CCA1364 and CCB1256 ${ }^{106} \mathrm{Ru}$ plaques, whose actual emitter distributions were measured. The idealized, homogeneous CCA and CCB plaques are also simulated. Results: The largest discrepancy in depth dose distribution observed between the heterogeneous and the homogeneous plaques was 7.9 and $23.7 \%$ for the CCA and CCB plaques, respectively. In terms of isodose lines, the line referring to $100 \%$ of the reference dose penetrates 0.2 and $1.8 \mathrm{~mm}$ deeper in the case of heterogeneous CCA and CCB plaques, respectively, with respect to the homogeneous counterpart. Conclusions: The observed differences in absorbed dose distributions obtained from heterogeneous and homogeneous plaques are clinically ir-
\end{abstract}

\section{KARGER}

(c) 2017 S. Karger AG, Basel

E-Mail karger@karger.com

www.karger.com/oop relevant if the plaques are used with a lateral safety margin of at least $2 \mathrm{~mm}$. However, these differences may be relevant if the plaques are used in eccentric positioning.

(c) 2017 S. Karger AG, Basel

\section{Introduction}

The determination of absorbed dose distributions from ruthenium eye applicators and the results for uveal melanoma have been the object of study during the last 40 years [1-8]. Initially, some researchers measured the absorbed dose distribution using different methods such as ionization chambers, diamond detectors, or thermoluminescent dosimeters. Later, a point-kernel numerical method was used to calculate the dose distribution around a curved radiation source with the shape of a spherical shell [9]. Other researchers combined the point-kernel and the Monte Carlo method [10,11]. Finally, some studies have used Monte Carlo algorithms to simulate the radiation transport produced by eye applicators to determine the dose distribution [11-17]. Until very recently, the commonly used treatment planning system for ${ }^{106} \mathrm{Ru}$ plaques was based on simplified physical models of radiation transport where the emitter substance was as- 
sumed to be homogeneously distributed over the surface of the plaques and the anatomy of the eye was approximated to a homogeneous water sphere. The latest version of Plaque Simulator (version 6.4.3) allows taking into account the measurements provided by the certificate of the plaque, but radiation transport is still performed using analytical methods (http://www.eyephysics.com/PS/ index.html).

The Monte Carlo method is generally accepted as the most accurate approach for computing absorbed dose distributions in radiotherapy patients. This is particularly true in the presence of small radiation fields, like the ones used for eye irradiation, where radiation transport cannot be accurately handled by the conventional nonstochastic algorithms usually employed in treatment planning systems. Despite the accuracy of the Monte Carlo method, albeit limited by the underlying cross section models and by the statistical uncertainty inherent in the method, calculations of dose distributions are still difficult $[11,14]$. Most published articles consider the radioactive substance to be homogeneously distributed, although actual eye plaques present inhomogeneous distributions of the emitter substance. Hot spots in which the activity exceeds the average of the plaque by as much as $25 \%$ are not unusual (e.g., in the case of the CCB1256 plaque presented herein). Due to the manufacturing process there are no 2 identical plaques.

In this context, the purpose of this work is to present a Monte Carlo simulation of 2 specific plaques considering the actual distribution of the radioactive substance. We compare the absorbed doses produced by 2 inhomogeneous plaques (specifically CCA1364 and CCB1256) with their homogeneous approximations.

\section{Materials and Methods}

The eye plaques considered were the models $\mathrm{CCA}$ and $\mathrm{CCB}$ from the manufacturer BEBIG GmbH (Berlin, Germany). These plaques are shaped like spherical caps. Both plaques have the same inner radius, measuring $1.2 \mathrm{~cm}$ along the symmetry axis. The significant geometric difference between the 2 models stems from the outer diameters of the caps across the rim, being $1.55 \mathrm{~cm}$ for the CCA model and $2.02 \mathrm{~cm}$ for the CCB model. For both plaques, the cap is $0.1 \mathrm{~cm}$ thick and is divided into 3 layers. The thicknesses of these layers from the inner to the outer surface of the cap are 0.01 , 0.02 , and $0.07 \mathrm{~cm}$. All layers are made of silver, with the middle layer containing the radioactive material.

The emitter substance is electrolytically deposited over the inner surface of the middle layer. The emitter substance does not cover the whole layer; it only extends to $0.07 \mathrm{~cm}$ from the cap rim. The active layer is encapsulated between the inner and the outer layer. The geometric computer models of the eye plaques were considered to be inside a spherical water phantom $6 \mathrm{~cm}$ in diameter. The focus of the plaques coincided with the center of the water phantom. The geometric descriptions of the plaques were accurately modeled using the constructive quadric geometry package provided by PENELOPE [18].

For each eye plaque, 2 radiation sources were considered: one in which the distribution of the emitter substance was homogeneous and another in which the heterogeneous experimental map was used. The emitter substance distributions for the CCA1364 and the CCB1256 ${ }^{106} \mathrm{Ru}$ plaque from Essen University Hospital were measured with a device developed at TU Dortmund University. This device uses a scintillation detector and a guiding system to measure the dose rate profile over the plaque's surface at a constant, small distance [19]. The process guarantees a high density of measured points and gapless coverage of the surface of the applicator. Both emitter dose maps were converted into normalized probability distributions that were used to model the radiation source using a code developed to this purpose. Figure la shows that the experimental emitter dose map for the CCA1364 plaque is heterogeneous with some hot and some cold areas. Figure $1 \mathrm{~b}$ shows the emitter map for the CCB1256 plaque, in which a large hot spot appears. The largest heterogeneity of the CCA plaque was about $18 \%$, while the largest heterogeneity of the $\mathrm{CCB}$ plaque amounted to $25 \%$. The accuracy of our simulation system for eye plaques had previously been validated by comparison of our simulated data in a water phantom with experimental results $[16,20]$.

${ }^{106} \mathrm{Ru}$ is a radioactive isotope that disintegrates into ${ }^{106} \mathrm{Rh}$, producing a beta spectrum characterized by a maximum energy of 39 $\mathrm{keV}$ and a half-life of 368 days. ${ }^{106} \mathrm{Rh}$ decays into stable ${ }^{106} \mathrm{Pd}$, producing a beta spectrum too. This last disintegration is of therapeutic interest and is characterized by a maximum energy of 3.540 $\mathrm{MeV}$ and a half-life of $29.8 \mathrm{~s}$. Simulations were run with the Monte Carlo general-purpose radiation transport code PENELOPE $[18,21]$ using penEasy $[22]$ as the main steering program.

penEasy is prepared neither for simulating a spectrum resulting from a beta disintegration nor for simulating a heterogeneous source distribution shaped as a spherical cap; therefore, the code was modified in 2 ways. The first modification consisted of simulating the decay of ${ }^{106} \mathrm{Rh}$ into ${ }^{106} \mathrm{Pd}$ through the 5 disintegrations with the highest yields, i.e., $3.540 \mathrm{MeV}$ (78.6\%), $3.050 \mathrm{MeV}$ (8.1\%), $2.410 \mathrm{MeV}$ (10.0\%), $2.000 \mathrm{MeV}$ (1.77\%), and $1.539 \mathrm{MeV}(0.46 \%)$. The endpoint energy was chosen at random according to the probabilities given by the yields for each primary particle sampled. The initial electron energies were then sampled at random from the corresponding beta decay spectrum. For that purpose, an adapted version of the EFFY code [23], which uses the Fermi theory to describe beta decay, was incorporated into the modified penEasy code.

The second modification allowed penEasy to use the emitter maps obtained from the measuring device as explained above. For each primary particle sampled, a position and a flight direction over the spherical layer containing the emitter substance were chosen at random according to the probabilities given by the geometric distribution of the spherical cap. Afterwards, an emission probability was sampled using a normalized, uniformly distributed random number. The primary particle sampled was accepted only if the sampled, uniformly distributed random number was lower than the normalized experimental distribution yielded for the region where the particle had previously been positioned; otherwise, the particle was rejected. 


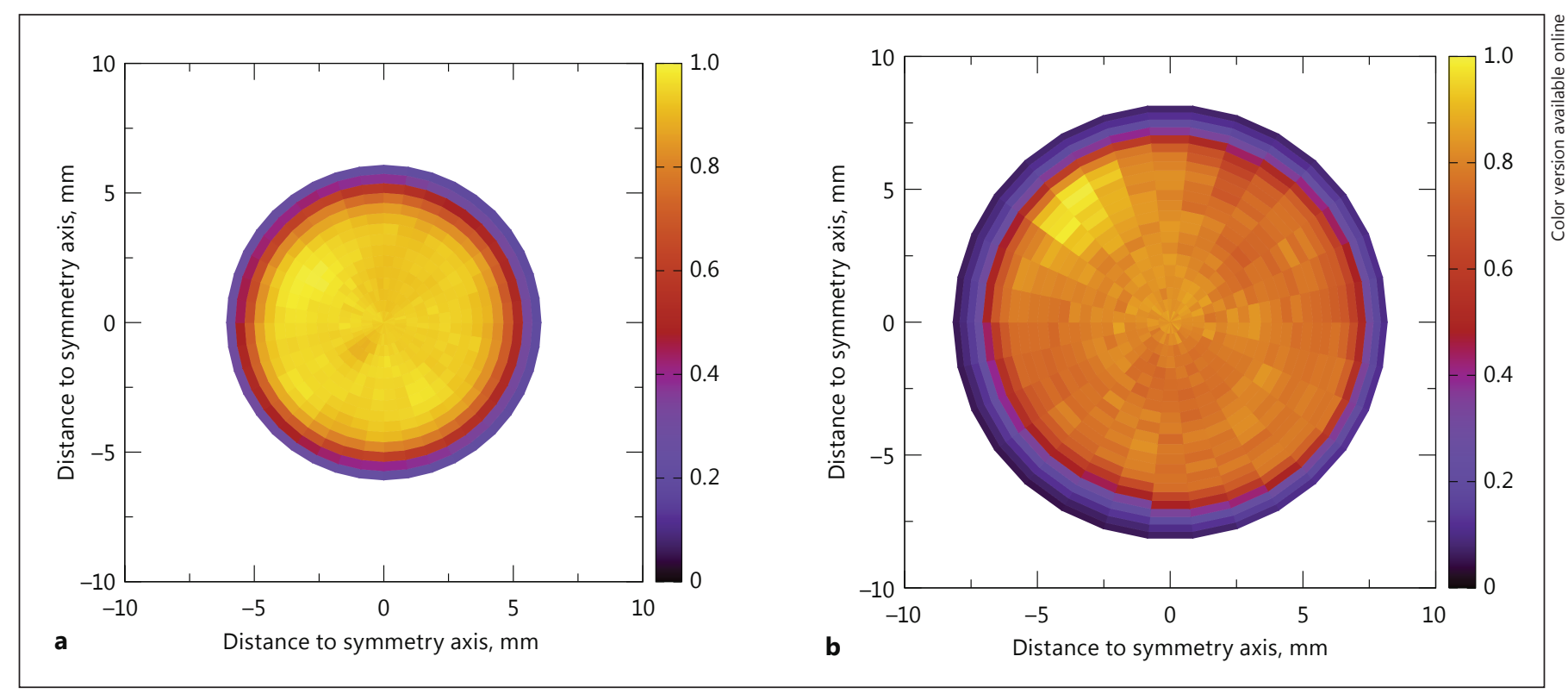

Fig. 1. Normalized experimental surface distribution of the emitter substance into the eye plaque. a CCA1364 eye plaque. b CCB1256 eye plaque.
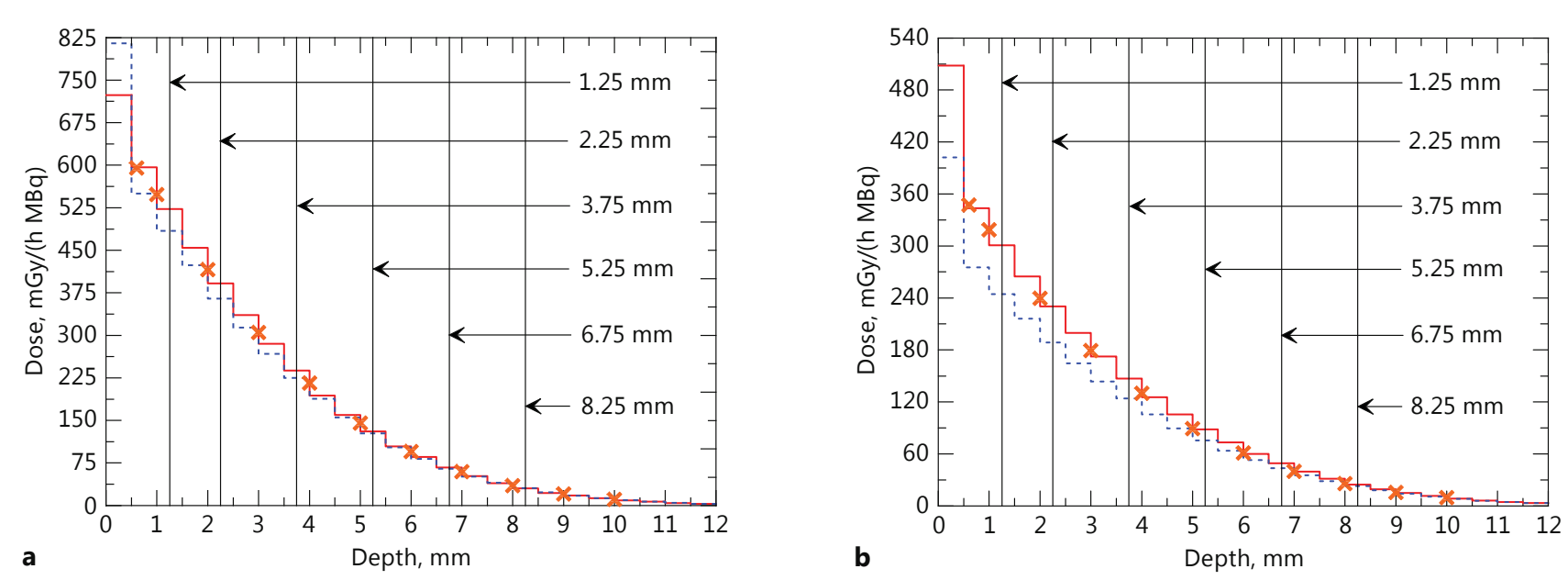

Fig. 2. Depth-dose comparison along the symmetry axis of the plaque. The plot represents the simulated ideal homogeneous distribution (dashed line), the simulated realistic heterogeneous distribution (continuous line), and the manufacturer experimental data (crosses). The standard statistical uncertainty of the simulated data is smaller than the thickness of the lines. a CCA1364 eye plaque. b CCB1256 eye plaque.

\section{Results and Discussion}

The depth dose distribution tallied for the CCA1364 heterogeneous plaque (Fig. 2a) differs from that of the homogeneous CCA plaque by $7.9 \%$ at a depth of $2 \mathrm{~mm}$. The depth is measured from the inner surface of the plaque along its symmetry axis. The heterogeneous dose distribution is statistically compatible with the measurements made by the manufacturer reproduced in the plaque certificate. The vertical lines plotted on Figure 2a correspond 

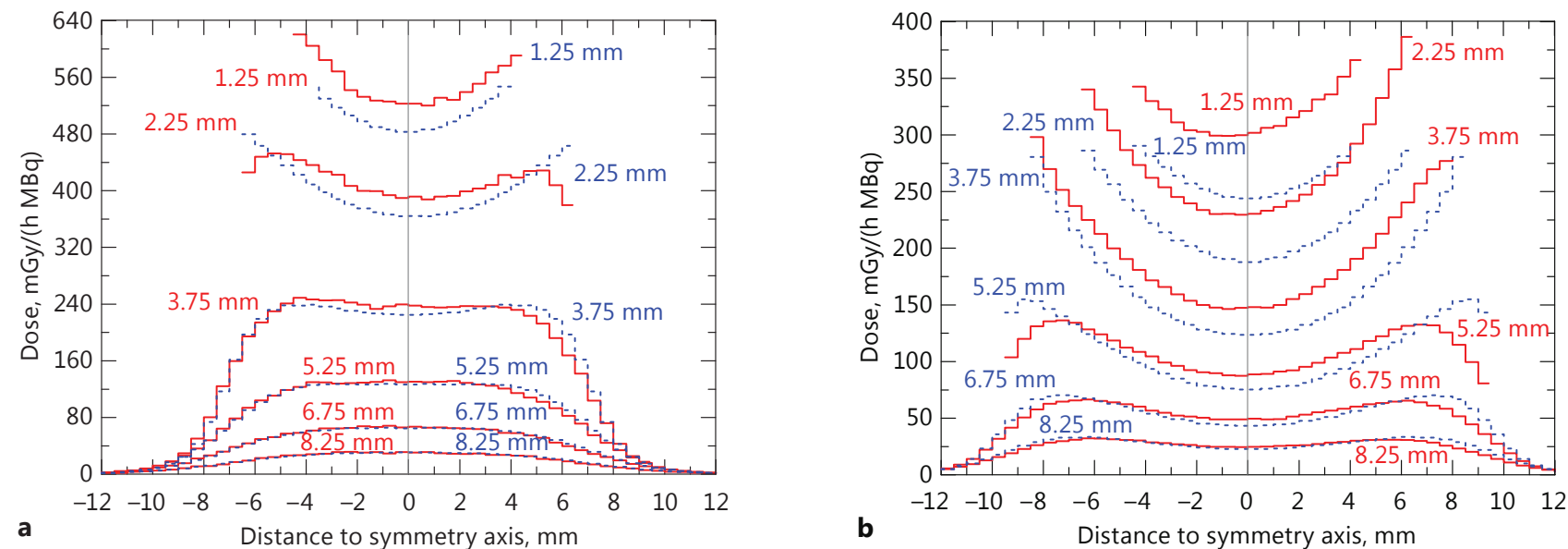

Fig. 3. Lateral profile perpendicular to the symmetry axis of the plaque. The plot represents the simulated ideal homogeneous distribution (dashed lines) and a simulated realistic heterogeneous

distribution (continuous lines). The standard statistical uncertainty of the simulated data is smaller than the thickness of the lines. a CCA1364 eye plaque. b CCB1256 eye plaque.
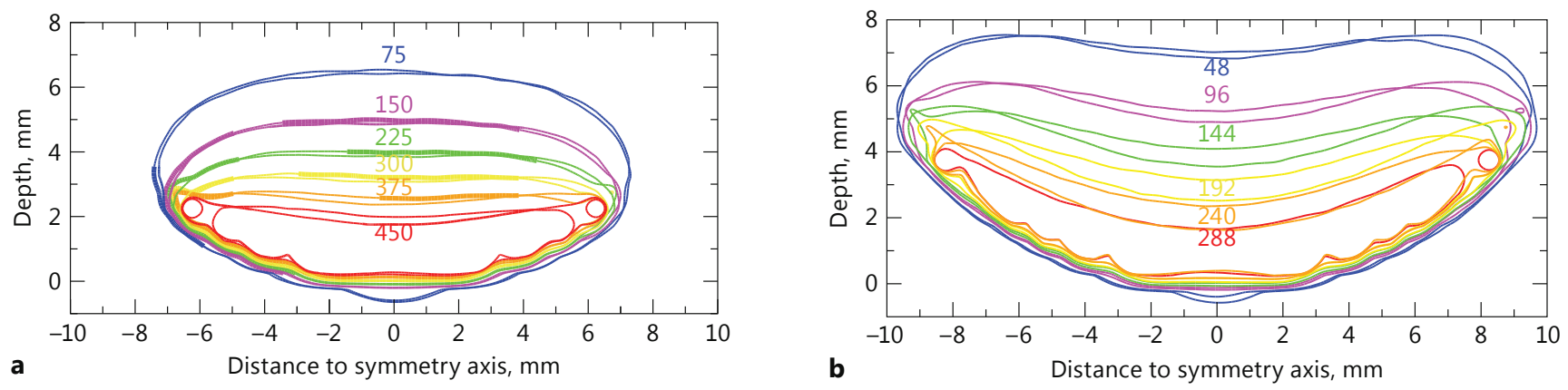

Fig. 4. Thin lines correspond to homogeneous emitter distribution. Thick lines correspond to heterogeneous emitter distribution. Isodose lines are plotted taking the dose measured by the manufacturer at a depth of $2 \mathrm{~mm}$ from the plaque surface as a reference. a CCA1364 isodose lines. Isodoses at 450, 375, 300, 225, 150 , and $75 \mathrm{mGy} /(\mathrm{h} \mathrm{MBq})$ correspond to $120,100,80,60,40$, and
$20 \%$ of the reference value, respectively. b CCB1256 isodose lines. Isodose lines at $288,240,192,141,96$, and $48 \mathrm{mGy} /(\mathrm{h} \mathrm{MBq})$ correspond to $120,100,80,60,40$, and $20 \%$ of the reference value, respectively. The isodose line of $288 \mathrm{mGy} /(\mathrm{h} \mathrm{MBq})$ is plotted exclusively for heterogeneous emitter distribution. to the depths where the lateral profiles shown in Figure $3 \mathrm{a}$ are estimated.

As may be observed in Figure 3a, the differences in lateral profiles between the inhomogeneous CCA1364 and the homogeneous CCA plaque are relevant near the symmetry axis. Regarding each depth, the differences along the symmetry axis are $8.2 \%$ observed on the lateral profile estimated at $1.25 \mathrm{~mm}, 7.5 \%$ at $2.25 \mathrm{~mm}, 5.9 \%$ at $3.75 \mathrm{~mm}, 3.1 \%$ at $5.25 \mathrm{~mm}, 3.9 \%$ at $6.75 \mathrm{~mm}$, and $0.7 \%$ at $8.25 \mathrm{~mm}$.

Figure 4a shows the differences between the isodose lines computed for the heterogeneous and homogeneous 
plaques. The discrepancies diminish with increasing distance from the plaque surface. The isodose lines from the homogeneous and the inhomogeneous plaque become very similar for a value of $225 \mathrm{mGy} /(\mathrm{h} \mathrm{MBq})$, that is, at $60 \%$ of the reference dose measured by the manufacturer. The difference in maximum depth to which each isodose is delivered is another piece of information given by the plot. The difference in depth between the homogeneous and the heterogeneous isodose lines corresponding to $450 \mathrm{mGy} /(\mathrm{h} \mathrm{MBq})$ and $375 \mathrm{mGy} /(\mathrm{h} \mathrm{MBq})$ is $0.2 \mathrm{~mm}$, while for $300 \mathrm{mGy} /(\mathrm{h} \mathrm{MBq})$ and $225 \mathrm{mGy} /(\mathrm{h} \mathrm{MBq})$ it is $0.1 \mathrm{~mm}$.

The heterogeneous CCB1256 plaque produces a dose distribution whose difference from that of the homogeneous CCB plaque amounts to $22 \%$ at the reference depth of $2 \mathrm{~mm}$ from the surface along the symmetry axis in the depth dose profile. The experimental depth dose distribution measured by the manufacturer shows good agreement with the results of the simulated inhomogeneous plaque, as shown in Figure 2b. The vertical lines correspond to the depths at which the lateral profiles are estimated.

For the CCB1256 plaque, the differences between the homogeneous and heterogeneous configurations are relevant for all lateral profiles (Fig. 3b). The differences are significant even far from the plaque surface, at $6.75 \mathrm{~mm}$. For each depth, the difference along the symmetry axis is $23.7 \%$ as observed on the lateral profile estimated at 1.25 $\mathrm{mm}$. For the rest of the lateral profiles tallied, the differences along the symmetry axis are $22.7 \%$ at $2.25 \mathrm{~mm}$, $19.6 \%$ at $3.75 \mathrm{~mm}, 17.8 \%$ at $5.25 \mathrm{~mm}, 14.4 \%$ at $6.75 \mathrm{~mm}$, and $8 \%$ at $8.25 \mathrm{~mm}$.

Figure $4 \mathrm{~b}$ shows the differences between the homogeneous and heterogeneous isodose lines. For the homogeneous plaque, the isodose line corresponding to $240 \mathrm{mGy} /$ (h MBq) (the reference dose obtained by the manufacturer at $2 \mathrm{~mm}$ ) coincides with the heterogeneous isodose line corresponding to $288 \mathrm{mGy} /(\mathrm{h} \mathrm{MBq})(120 \%$ of the reference dose). The discrepancies are reduced with increasing distance from the plaque surface, and the isodose lines from both the homogeneous and the heterogeneous plaque are compatible within the statistical uncertainty for a value of $48 \mathrm{mGy} /(\mathrm{h} \mathrm{MBq})$, that is, at $20 \%$ of the reference dose. The difference in depth between the homogeneous and the heterogeneous isodose line corresponding to $240 \mathrm{mGy} /(\mathrm{h} \mathrm{MBq})$ is $1.8 \mathrm{~mm}$; for the $192 \mathrm{mGy} /(\mathrm{h}$ $\mathrm{MBq}$ ) isodose line it is $0.6 \mathrm{~mm}$, for the $144 \mathrm{mGy} /(\mathrm{h} \mathrm{MBq})$ isodose line it is $0.6 \mathrm{~mm}$, for the $96 \mathrm{mGy} /(\mathrm{h} \mathrm{MBq})$ isodose line it is $0.3 \mathrm{~mm}$, and for the $48 \mathrm{mGy} /(\mathrm{h} \mathrm{MBq})$ isodose line it is $0.2 \mathrm{~mm}$.

\section{Conclusions}

The experimental data obtained with the device developed at TU Dortmund University were used to model the distribution of the emitter substance in 2 specific heterogeneous ruthenium eye plaques. The dose distributions obtained by Monte Carlo simulation of heterogeneous plaques matched the experimental data provided by the manufacturer in the plaque certificates. For the CCA1364 plaque, differences between heterogeneous and homogeneous emitter distribution are relevant at distances smaller than $3.75 \mathrm{~mm}$. For the CCB1256 plaque, the emitter distribution presents inhomogeneities of about $25 \%$ compared with the ideal homogeneous plaque. These inhomogeneities translated into discrepancies in dose distribution of nearly $25 \%$ between the heterogeneous and the idealized homogeneous plaque. For the CCB1256 plaque, differences between heterogeneous and homogeneous emitter distribution are relevant up to $6.75 \mathrm{~mm}$ from the plaque surface.

${ }^{106} \mathrm{Ru}$ plaques deliver high doses to a very restricted volume, thereby having a large therapeutic window. They are very effective for tumors with apical heights up to 4 $\mathrm{mm}$ and may be used by an experienced team for tumors up to $6 \mathrm{~mm}$ in height.

The differences in absorbed dose distributions using either the actual heterogeneous plaque or the idealized homogenous plaque are clinically irrelevant as long as the plaque covers the tumor symmetrically with a lateral safety margin of at least $2 \mathrm{~mm}$. However, these differences may lead to underdosage for eccentric positioning (see right side of Fig. 4b), which might be necessary for treating tumors at the posterior pole of the eye.

\section{Acknowledgements}

The authors are thankful to the Deutsche Forschungsgemeinschaft (projects BR 4043/3-1 and EI 869/1-3).

\section{Statement of Ethics}

The research presented herein does not involve studies on humans, animals, or biological materials.

\section{Disclosure Statement}

The authors do not have any conflict of interests. 


\section{References}

1 Tjho-Heslinga RE, Kakebeeke-Kemme HM, Davelaar J, et al: Results of ruthenium irradiation of uveal melanoma. Radiother Oncol 1993;29:33-38.

2 Tjho-Heslinga RE, Davelaar J, Kemme HM, et al: Results of ruthenium irradiation of uveal melanomas: the Dutch experience. Radiother Oncol 1999;53:133-137.

3 Mourtada F, Koch N, Newhauser W: ${ }^{106} \mathrm{Ru} /$ ${ }^{106} \mathrm{Rh}$ plaque and proton radiotherapy for ocular melanoma: a comparative dosimetric study. Radiat Prot Dosimetry 2005;116(pt 2): 454-460.

4 Schueler AO, Flühs D, Anastassiou G, et al: $\beta$-Ray brachytherapy with ${ }^{106} \mathrm{Ru}$ plaques for retinoblastoma. Int J Radiat Oncol Biol Phys 2006;65:1212-1221.

5 Finger PT: Radiation therapy for orbital tumors: concepts, current use, and ophthalmic radiation side effects. Surv Ophthalmol 2009; 54:545-568.

6 Russo A, Laguardia M, Damato B: Eccentric ruthenium plaque radiotherapy of posterior choroidal melanoma. Graefes Arch Clin Exp Ophthalmol 2012;250:1533-1540.

7 American Brachytherapy Society - Ophthalmic Oncology Task Force; ABS - OOTF Committee: The American Brachytherapy Society consensus guidelines for plaque brachytherapy of uveal melanoma and retinoblastoma. Brachytherapy 2014;13:1-14.

8 Takiar V, Voong KR, Gombos DS, et al: A choice of radionuclide: comparative outcomes and toxicity of ruthenium-106 and iodine-125 in the definitive treatment of uveal melanoma. Pract Radiat Oncol 2015;5:e169e176.
9 Hokkanen J, Heikkonen J, Holmberg P: Theoretical calculations of dose distributions for beta-ray eye applicators. Med Phys 1997;24: 211-213.

10 Davelaar J, Schaling DF, Hennen LA, Broerse JJ: Dosimetry of ruthenium-106 eye applicators. Med Phys 1992;19:691-694.

11 Astrahan MA: A patch source model for treatment planning of ruthenium ophthalmic applicators. Med Phys 2003;30:1219-1228.

12 Cross W, Hokkanen J, Järvinen H, et al: Calculation of beta-ray dose distributions from ophthalmic applicators and comparison with measurements in a model eye. Med Phys 2001;28:1385-1396.

13 Sánchez-Reyes A, Tello J, Guix B, Salvat F: Monte Carlo calculation of the dose distributions of two ${ }^{106} \mathrm{Ru}$ eye applicators. Radiother Oncol 1998;49:191-196.

14 Hermida-López M: Calculation of dose distributions for $12{ }^{106} \mathrm{Ru} /{ }^{106} \mathrm{Rh}$ ophthalmic applicator models with the PENELOPE Monte Carlo code. Med Phys 2013;40:101705.

15 Barbosa NA, Ribeiro LA, de Menezes AF, et al: Assessment of ocular beta radiation dose distribution due to ${ }^{106} \mathrm{Ru} /{ }^{106} \mathrm{Rh}$ brachytherapy applicators using MCNPX Monte Carlo code. Int J Cancer Ther Oncol 2014;2:02038.

16 Brualla L, Sempau J, Zaragoza FJ, et al: Accurate estimation of dose distributions inside an eye irradiated with ${ }^{106} \mathrm{Ru}$ plaques. Strahlenther Onkol 2013;189:68-73.
17 Brualla L, Zaragoza FJ, Sauerwein W: Monte Carlo simulation of the treatment of eye tumors with ${ }^{106} \mathrm{Ru}$ plaques: a study on maximum tumor height and eccentric placement. Ocul Oncol Pathol 2014;1:2-12.

18 Salvat F, Fernández-Varea JM, Sempau J: PENELOPE - A Code System for Monte Carlo Simulation of Electron and Photon Transport. Issy-les-Moulineaux, OECD Nuclear Energy Agency, 2011.

19 Eichmann M, Flühs D, Spaan B: Development of a high precision dosimetry system for the measurement of surface dose rate distribution for eye applicators. Med Phys 2009;36:46344643.

20 Brualla L, Sempau J, Sauerwein W: Comment on "Monte Carlo calculation of the dose distributions of two ${ }^{106} \mathrm{Ru}$ eye applicators" [Radiother Oncol 49 (1998) 191-196]. Radiother Oncol 2012;104:267-268.

21 Sempau J, Acosta E, Baró J, et al: An algorithm for Monte Carlo simulation of coupled electron-photon transport. Nucl Instrum Methods Phys Res B 1997;132:377-390.

22 Sempau J, Badal A, Brualla L: A PENELOPEbased system for the automated Monte Carlo simulation of clinacs and voxelized geometries - application to far-from-axis fields. Med Phys 2011;38:5887-5895.

23 García-Toraño E, Grau Malonda A: EFFY, a new program to compute the counting efficiency of beta particles in liquid scintillators. Comput Phys Commun 1985;36:307-312. 